\title{
Waveform Distortion of Gaussian Beam in Atmospheric Turbulence Simulated by Phase Screen Method
}

\author{
Zhi-Qiang Yang $\mathbb{D}^{1},{ }^{1}$ Li-hong Yang, ${ }^{1}$ Lei Gong, ${ }^{1}$ Liguo Wang, ${ }^{1}$ and Xinyi Wang ${ }^{2}$ \\ ${ }^{1}$ School of Opto-electronic Engineering, Xi'an Technological University, Xi'an 710021, China \\ ${ }^{2}$ Department of Engineering, Queens' College, University of Cambridge, Cambridge, UK \\ Correspondence should be addressed to Zhi-Qiang Yang; yangzhiqiang@xatu.edu.cn
}

Received 30 November 2021; Accepted 20 January 2022; Published 21 February 2022

Academic Editor: Heng Liu

Copyright (C) 2022 Zhi-Qiang Yang et al. This is an open access article distributed under the Creative Commons Attribution License, which permits unrestricted use, distribution, and reproduction in any medium, provided the original work is properly cited.

\begin{abstract}
The atmospheric turbulence phase screen is generated based on the power spectrum inversion method, and the multiple transmission processes are statistically averaged. The waveform distortion of the Gaussian beam in the atmospheric turbulence is analyzed; the simulation results show that the property of Gaussian beam has been destroyed after its passing through the atmospheric turbulence. When the beam waist radius is close to a certain radius, the degree of change becomes larger. When it approaches the critical value, the wave surface no longer changes sharply, and as the turbulence intensity increases, the phase fluctuation becomes more and more severe, the coherence of the beam is destroyed, and the spot may be split into several pieces. Finally, the relationship of intensity fluctuation, amplitude fluctuation, and bit error rate with distance is analyzed.
\end{abstract}

\section{Introduction}

The particularity of light propagation in turbulent media lies in the random fluctuation of the refractive index of the medium; the key of numerical simulation for laser atmospheric propagation is to construct a phase screen that can correctly reflect the statistical characteristics of atmospheric turbulence [1-4]. The earliest phase screen simulation turbulence method is proposed by Fleck, who used the "multiphase screen method" in 1976 to simulate the influence of atmospheric turbulence in free space on the transmitted beam. This method is widely used to simulate the atmospheric turbulence effect of the beam [5]. The current methods of generating phase screens are mainly to filter Gaussian white noise by using the refractive index power spectrum of the turbulence, by which the disturbed wavefront phase can be obtained through Fourier transform; this method is called "power spectrum inversion method." Since Mcglamery proposed the power spectrum inversion method in 1967, this method has been widely used to simulate turbulence [6]. In 1976, Noll proposed to use orthogonal and complete two-dimensional basis functions (such as Zenike polynomials and K-L polynomials) to directly obtain the distorted wavefront of turbulence [7]. In 1983, Walner proposed a method of using the covariance of the phase structure function to generate the Kolmogorov turbulence phase screen [8], but this method is computationally expensive. In 1990, Bowman proposed an algorithm for reconstructing the turbulent wavefront using wavelet transform [9]. Among many methods, the power spectrum inversion method is applicable to a wide range of atmospheric turbulence spectrum models [10-12].

Based on the Gaussian beam horizontal propagation theory, this paper uses MATLAB to carry out detailed simulation on the power spectrum inversion method and analyzes the simulation results. The propagation characteristics of the Gaussian beam under the atmospheric turbulence spectrum are discussed. 


\section{Theoretical Model of Laser Transmission in Turbulent Atmosphere}

Assuming that the monochromatic electromagnetic wave is transmitted along the $Z$ direction, the wave field equation is

$$
\vec{U}(x, y, z, t)=U(x, y, z) \exp [-i(\omega t-k z)],
$$

where $\omega$ is the frequency of monochromatic electromagnetic waves. When it propagates in the atmosphere (nonmagnetic medium $\mu=1)$ without free charge $\left(\rho_{0}=0\right)$ and free current $\left(J_{0}=0\right)$, its wave equation can be expressed as

$$
\nabla^{2} U(x, y, z, t)+k^{2} n(\vec{r})^{2} U(x, y, z, t)=0,
$$

where $n(\vec{r})$ is a function of $\vec{r}$ which represents the local refractive index at this point. It is very difficult to accurately solve this basic equation of light transmission in atmosphere.

By removing the vector operator, the equation can be turned into a scalar equation:

$$
2 i k \frac{\partial U(x, y, z)}{\partial z}+\nabla^{2} U(x, y, z)+2 k^{2} n_{1}(r) U(x, y, z)=0
$$

where $U(x, y, z)$ is a slowly varying function of $z$; it can only change at the distance of random medium scale $l$, so as long as $l>>\lambda$; then, $\left|\partial^{2} U / \partial z^{2}\right| \ll 2 \kappa|\partial U / \partial z|$, and $2 n_{1}(\vec{r})$ approximately replaces $2 n_{1}(\vec{r})+n_{1}^{2}(\vec{r})\left(n_{1}(\vec{r})\right.$ represents the fluctuation of atmospheric refractive index at position $\vec{r}$ ). By simplifying formula (3), the following formula can be obtained:

$$
2 i k \frac{\partial U(x, y, z)}{\partial z}+\nabla_{\perp}^{2} U(x, y, z)+2 k^{2} n_{1}(r) U(x, y, z)=0
$$

Equation (4) is called the parabolic equation or quasioptical approximation relationship, which is suitable for the propagation of narrow-angle expanded beams. $k$ is equal to $2 \pi / \lambda$ which represents wave number in free space, and $\nabla_{\perp}^{2}=\left(\partial^{2} / \partial x^{2}\right)+\left(\partial^{2} / \partial y^{2}\right)$. The infinitely extending random medium can be divided into many sections with thickness of $\Delta z$; the influence of turbulence in each $\Delta z$ section on the phase of light waves can be concentrated on a thicknessless phase screen, but this phase screen has no effect on the amplitude of the light wave. The change of light wave amplitude is the cumulative result of free-space diffraction at the $\Delta z$ distance between many two-phase screens.

In order to specify each random phase screen, the correlation function and power spectrum of the phase fluctuation on each phase screen can be calculated. Assuming that there is no diffraction on each phase screen, from the perspective of geometric optics, the correlation function of the phase fluctuation can be written:

$$
\Gamma_{\theta}\left(\vec{\rho}_{1}, \vec{\rho}_{2}\right)=k^{2} \int_{0}^{\Delta z} \int_{0}^{\Delta z}\left\langle n_{r}\left(z_{1}, \vec{\rho}_{1}\right) n_{1}\left(z_{2}, \vec{\rho}_{2}\right)\right\rangle \mathrm{d} z_{1} \mathrm{~d} z_{2}=k^{2} \Delta z \Gamma_{n}\left(\vec{\rho}_{1}-\vec{\rho}_{2}\right)
$$

where $\vec{\rho}$ is the vector in the coordinate plane perpendicular to the $z$ direction (the $x y$ plane), $\Gamma_{\theta}(\vec{\rho})$ is the correlation function of the phase fluctuation, and $\Gamma_{n}(\vec{\rho})$ is the correlation function of refractive index fluctuation. Assuming $A z>\rho_{0}$ ( $\rho_{0}$ is the coherence length of the turbulence), formula (5) can be simplified:

$$
\Gamma_{n}(\vec{\rho})=2 \pi \int_{-\infty}^{\infty} \int_{-\infty}^{\infty} \phi_{n}\left(\vec{k}_{\perp}, k_{z}=0\right) \exp \left(i \vec{k}_{\perp} \cdot \vec{\rho}\right) \mathrm{d} \vec{k}_{\perp}
$$

So, the relationship between the power spectrum function and the power spectrum of the turbulent refractive index of each phase screen can be obtained:

$$
\phi_{\theta}\left(\vec{k}_{\perp}\right)=2 \pi k^{2} \Delta z \phi_{n}\left(\vec{k}_{\perp}, k_{z}=0\right)
$$

Because the transmission of the light wave field between two adjacent phase screens can be approximated as the transmission in free space, which represents the fluctuation of the refractive index $n_{1}(r)=0$, it can be obtained from equation (4):

$$
\frac{\partial U(x, y, z)}{\partial z}=\frac{i}{2 k} \nabla_{\perp}^{2} U(x, y, z) .
$$

It can be obtained by Fourier transform of two dimensional on both sides at the same time:

$$
\frac{\partial \widetilde{U}\left(\vec{k}_{\perp}, z\right)}{\partial z}=-\frac{i \vec{k}_{\perp}}{2 k} U\left(\vec{k}_{\perp}, z\right)
$$

where $\vec{k}_{\perp}$ is the wave vector perpendicular to the transmission direction; by integral in the $Z$ direction on both sides of equation (9), solution can be obtained:

$$
\partial \widetilde{U}\left(\vec{k}_{\perp}, z+\delta_{z}\right)=\widetilde{U}\left(\vec{k}_{\perp}, z\right) \exp \left[-i \frac{k_{\perp}^{2} \Delta z}{2 k}\right] .
$$

Equation (10) shows the free-space diffraction between the two-phase screens.

As shown in Figure 1, after replacing the infinitely extending turbulent medium with a large number of phase screens, the transmission of light waves through the turbulent atmosphere is equivalent to the transmission of light waves between phase screens after passing through the phase screens.

(a) The contribution of the phase screen to the phase of the light wave field is as follows:

$$
U\left(\vec{\rho}, z_{i}^{+}\right)=U\left(\vec{\rho}, z_{i}^{-}\right) \exp [i \theta(\vec{\rho})]
$$

where $z_{i}^{+}$represents the right side of the $i$ th phase screen and $z_{i}^{-}$represents the left side of the $i$ th phase screen. 


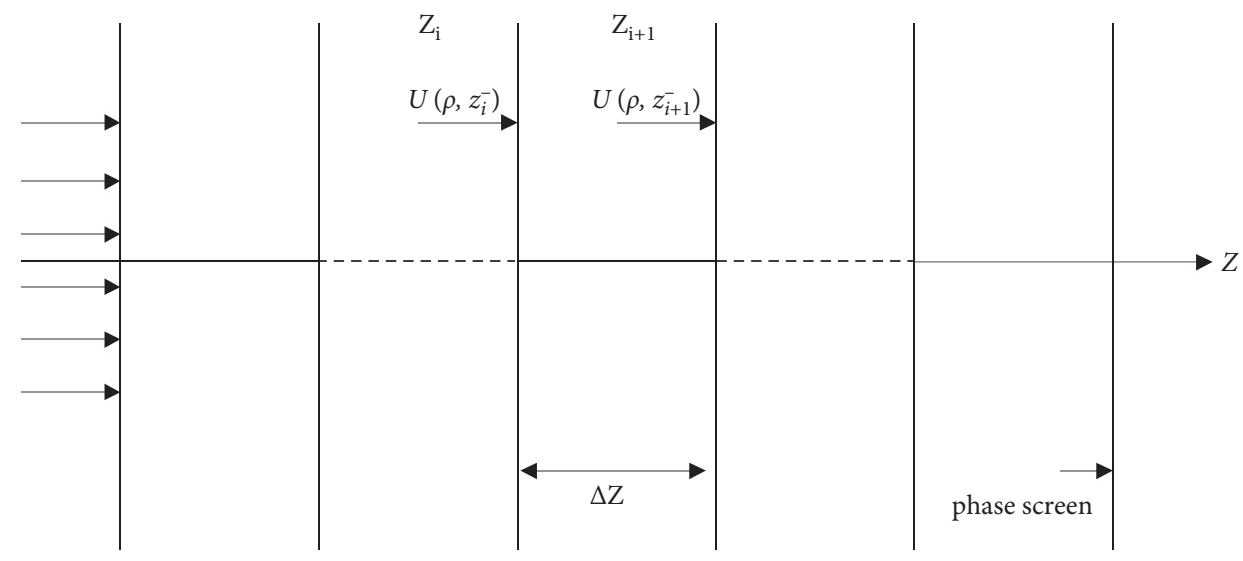

FIGURE 1: Schematic diagram of simulating the propagation of light waves in turbulent atmosphere with the phase screen approximation method.

(b) Perform Fourier transform on $U\left(\vec{\rho}, z_{i}^{+}\right)$to get $\widetilde{U}\left(k_{\perp}, z_{i}^{+}\right)$.

(c) Through the free-space transmission with a distance of $\Delta z$

$$
\widetilde{U}\left(\vec{k}_{\perp}, z_{i+1}^{-}\right)=\tilde{U}\left(\vec{k}_{\perp}, z_{i}^{+}\right) \exp \left[-i \frac{k_{\perp}^{2} \Delta z}{2 k}\right] .
$$

(d) By performing Fourier transform on $\widetilde{U}\left(\vec{k}_{\perp}, z_{i+1}^{+}\right)$, $U\left(\vec{\rho}, z_{i+1}^{-}\right)$can be obtained:

$$
U\left[\vec{\rho},(n+1) \Delta z^{-}\right]=F_{t}^{-1}\left\{F_{t}\left[U\left(\vec{\rho}, n \Delta z^{-}\right) \times \exp \left[i \theta_{n}(\vec{\rho})\right]\right] \exp \left(-\frac{i k_{\perp}^{2}}{2 k} \Delta z\right)\right\}
$$

Then, the transmission of light waves in the entire infinitely extending turbulent medium can be realized by repeatedly using equation (13) until the transmission of the last phase screen is completed.

\section{Numerical Simulation Method}

The parabolic equation is used to express the light field when the beam propagates in the turbulent atmosphere. The propagation path of the beam can be divided into two parts, the free space and the thin phase screen dispersed in it. When the light beam is transmitted to the phase screen, the wave front of the light is added with the corresponding disturbance part, and it passes through a section of free space to reach the next phase screen. When the beam reaches the receiving end, the entire simulation process ends. From equation (13), the light field at any adjacent phase screen can be expressed by the following formula $[13,14]$ :

$$
U\left(\vec{\rho}, z_{j+1}\right)=F_{t}^{-1}\left\{F_{t}\left\{U\left(\vec{\rho}, z_{j}\right) \exp \left[i \theta_{n}(\vec{\rho})\right]\right\} \exp \left[-i\left(\frac{k_{x}^{2}}{2 k}+\frac{k_{y}^{2}}{2 k}\right) \Delta z_{j+1}\right]\right\} .
$$

Equation (14) represents the turbulent phase screen, and $k_{x}$ and $k_{y}$ represent the wave number. The light wave transmission distance is divided into $N_{Z}$ sections, and the $j$ th phase screen is in $z_{j}, \Delta z_{j}$ is the phase screen spacing, and each phase screen is divided into $N \times N$ square grids whose grid spacing is $\Delta x$.

Power spectrum of the atmospheric turbulence is used to filter the complex Gauss random number matrix; by inverse
Fourier transform, the atmospheric disturbance phase will be obtained, which is the core of the power spectrum inversion method.

MATLAB is used to generate a complex Gaussian random number matrix which has a mean value of 0 and a variance of 1 , and then, the atmospheric refractive index power spectrum is introduced; the modified Von Karman spectrum is as follows: 


$$
\Phi_{n}(k)=0.033 C_{n}^{2} \exp \left(-\frac{k^{2}}{k_{m}^{2}}\right)\left(k^{2}+k_{0}^{2}\right)^{-11 / 6}, \quad 0<k<\infty
$$

The Kolmogorov spectrum is as follows:

$$
\Phi_{n}(\kappa, z)=0.033 C_{n}^{2}(z) \kappa^{-11 / 3},
$$

which is used to produce a continuous phase screen by filtering matrix $a$ with the atmospheric refraction power spectrum [14]:

$$
\phi(x, y)=C \sum_{k_{x}} \sum_{k_{y}} R\left(\kappa_{x}, \kappa_{y}\right) \sqrt{F_{\phi}\left(\kappa_{x}, \kappa_{y}\right)} e^{j\left(\kappa_{x} x+\kappa_{y} y\right)}
$$

$$
U(x, y)=-\frac{i}{\lambda z} \exp (i k z) \exp \left(i k \frac{x^{2}+y^{2}}{2^{z}}\right) \iint U_{0}\left(x_{0}+y_{0}\right) \times \exp \left(i k \frac{x^{2}+y^{2}}{2^{z}}\right) \times \exp \left[-i 2 \pi\left(f_{x_{0}}+f_{y_{0}}\right)\right] \mathrm{d} x_{0} \mathrm{~d} y_{0}
$$

where $f$ is the spatial frequency; by Fourier transform of $U_{0}\left(x_{0}+y_{0}\right) \exp \left(i k\left(\left(x_{0}^{2}+y_{0}^{2}\right) / 2\right)\right)$, the transmission in the vacuum part can be realized.

\section{Numerical Simulation Results}

The simulation parameters are set as follows: wavelength is $1.55 \mu \mathrm{m}$, number of sampling points is $256 \times 256$, phase screen width is $0.5 \mathrm{~m}$, phase screen spacing is $300 \mathrm{~m}$, and 1000 phase screens are constructed.

For the modified Von Karman spectrum, atmospheric turbulence phase screen for the different atmospheric refractive index structure constant and distortion of Gaussian beam after passing through the phase screen are simulated, and the results are as follows.

Figure 2 is the atmospheric turbulence phase screen generated by the power spectrum inversion method. Figures 2(a) to 2(f) are the atmospheric turbulence phase screens when the atmospheric refractive index structure constant $C_{n}^{2}$ takes different values. From figures, it is can be seen that the turbulence intensity increases gradually with the increase of atmospheric refractive index structure constant $C_{n}^{2}$.

Figure 3 shows the light intensity distribution diagram of the initial beam with the beam waist radius $w_{0}=4 \mathrm{~cm}$. Figure 3 shows Gaussian beam after passing through turbulence simulated by the phase screen theory, where Figures 4(a)-4(f) represent the phase fluctuation and light intensity distribution of the distorted Gaussian beam after passing through phase screens of different intensities.

Comparing Figures 4(a), 4(c), and 4(e) with Figure 3(a), after the Gaussian beam passes through atmospheric turbulence, the Gaussian property of the beam is destroyed. Compared with the initial Gaussian beam, the wave surface becomes rugged, the faster the change within the beam waist where $R$ represents the spatial spectrum of random function with normal distribution with mean value of 0 and variance of 1 and $F$ represents atmospheric turbulence power spectrum. $\Delta \kappa_{x}$ and $\Delta \kappa_{y}$ are the sampling interval, and the constant $C$ comes from the scale factor, which is used to adjust the phase screen variance. Fresnel diffraction is adopted in the vacuum transmission part, and the light field after the beam transmission distance $z$ can be expressed as [15]

radius, but when approaching a certain distance from the center, the wave surface no longer changes sharply but up and down changes. As the atmospheric refractive index structure constant increases, that is, the turbulence intensity increases, in fact, the three images correspond to weak turbulence, medium turbulence, and strong turbulence. When the beam waist radius is close to a certain radius, its degree of change keeps getting bigger. When it approaches the critical value, the wave surface no longer changes sharply, but as the distance is close to the center point, the greater the turbulence intensity, the greater the ups and downs of the wave surface. By comparing Figures 4(b), 4(d), and 4(f) with Figure 3(b), it can be obtained after the Gaussian beam passing through the atmospheric turbulence phase screen which is generated by the spectrum inversion method; the spot becomes blurred and scattered, and the focusing ability becomes worse, which indicates the phase of the Gaussian beam fluctuates. With the increase of $C_{n}^{2}$, the intensity of atmospheric turbulence continues to increase, the phase fluctuation becomes more and more severe, the coherence of the beam is destroyed, and the light spot may be split into several pieces. Compared with the initial Gaussian beam, the intensity of the beam attenuates significantly, and the larger the $C_{n}^{2}$, the greater the attenuation of the light intensity.

\section{The Effect of Turbulent Atmosphere on Free- Space Optical Communications}

In Free-Space Optical Communications (FSO) [16, 17], the laser from the transmitting end can be treated as a plane wave after being collimated by an optical lens [18, 19]; for the plane wave,

$$
\left\langle x^{2}\right\rangle=\sigma_{x}^{2}=0.31 C_{n}^{2} k^{7 / 6} L^{11 / 6}
$$




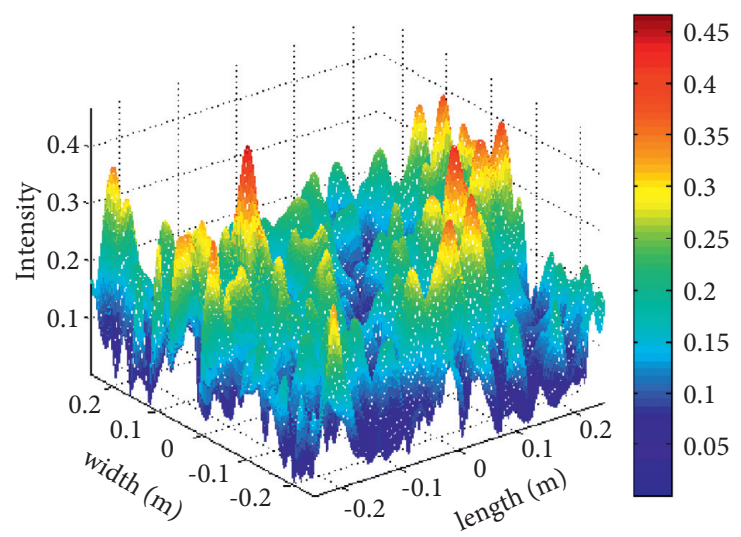

(a)

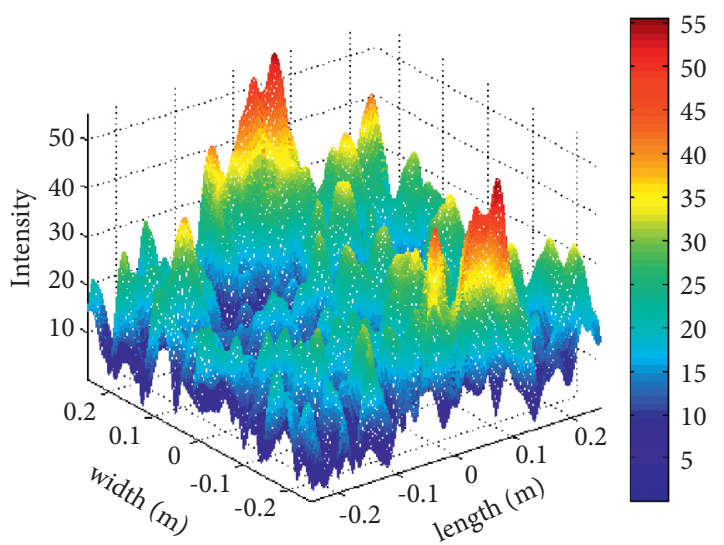

(c)

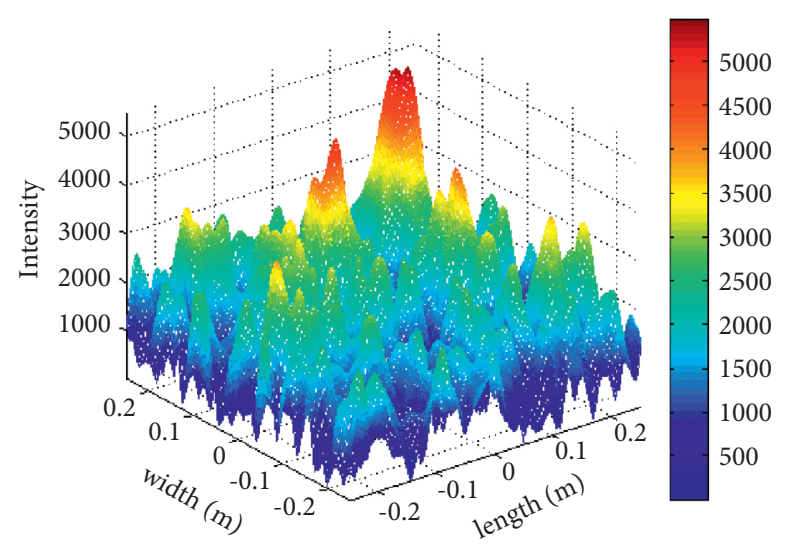

(e)

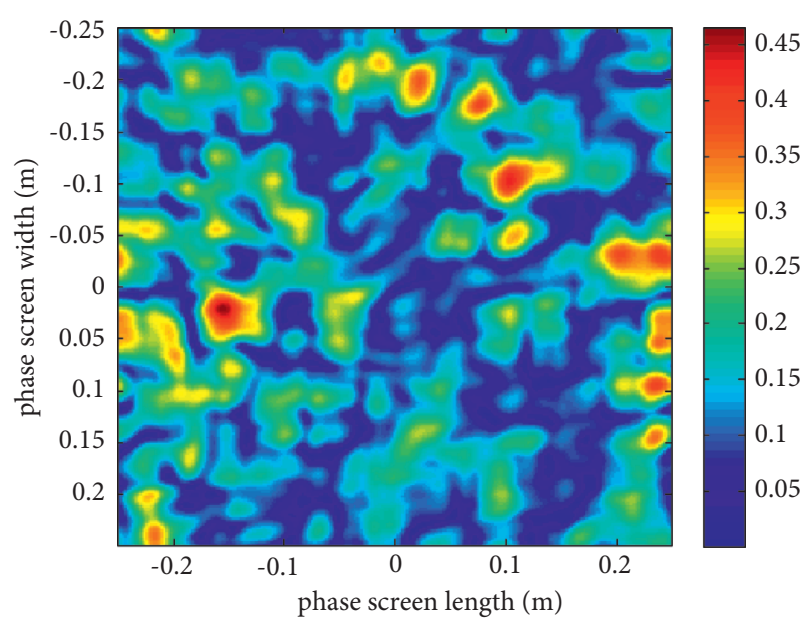

(b)

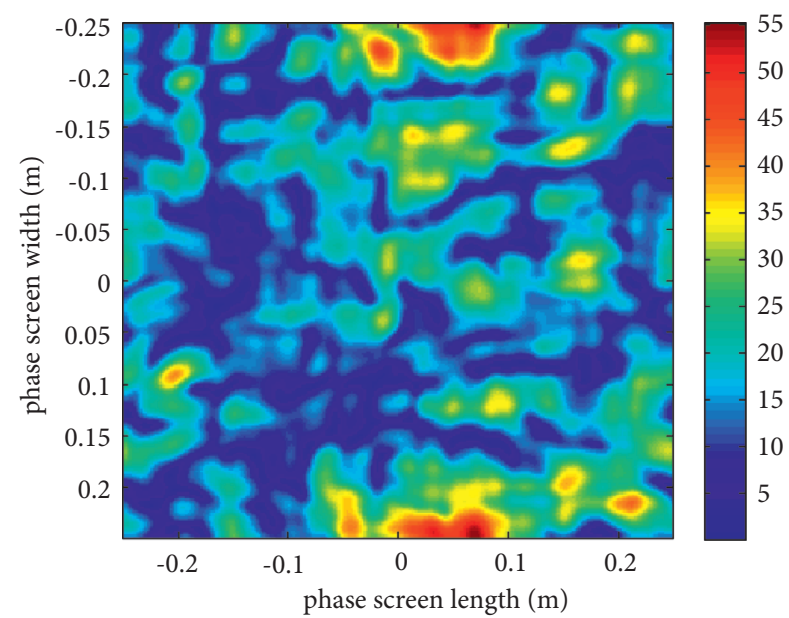

(d)

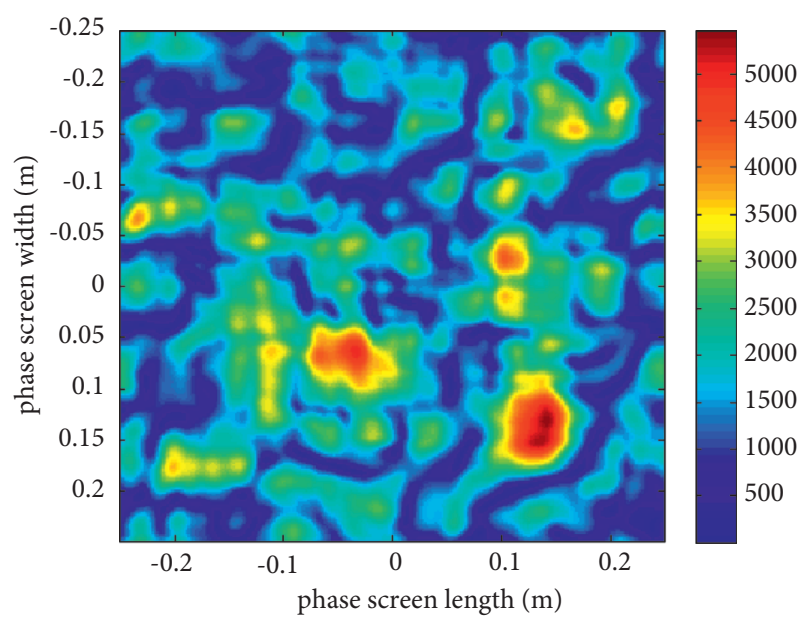

(f)

FIGURE 2: Atmospheric turbulence phase screen for the different atmospheric refractive index structure constants $C_{n}^{2}$. (a) $C_{n}^{2}=2 \times 10^{-17} \mathrm{~m}^{-2 / 3}$, (b) $C_{n}^{2}=2 \times 10^{-17} \mathrm{~m}^{-2 / 3}$, (c) $C_{n}^{2}=2 \times 10^{-15} \mathrm{~m}^{-2 / 3}$, (d) $C_{n}^{2}=2 \times 10^{-15} \mathrm{~m}^{-2 / 3}$, (e) $C_{n}^{2}=2 \times 10^{-13} \mathrm{~m}^{-2 / 3}$, and $(\mathrm{f})$ $C_{n}^{2}=2 \times 10^{-13} \mathrm{~m}^{-2 / 3}$. 


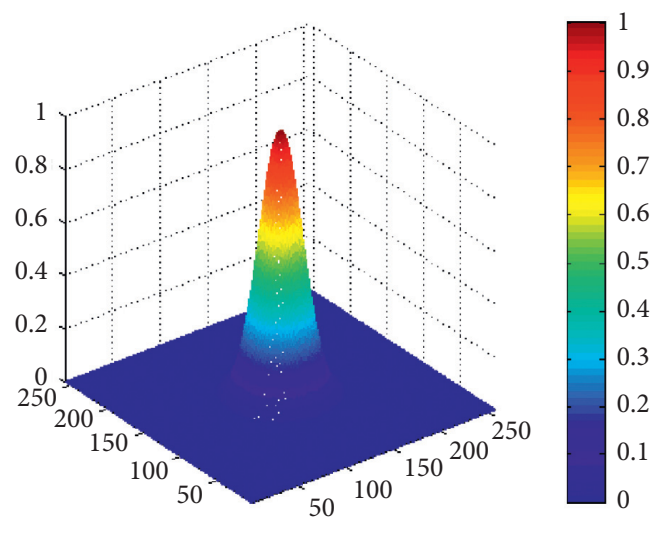

(a)

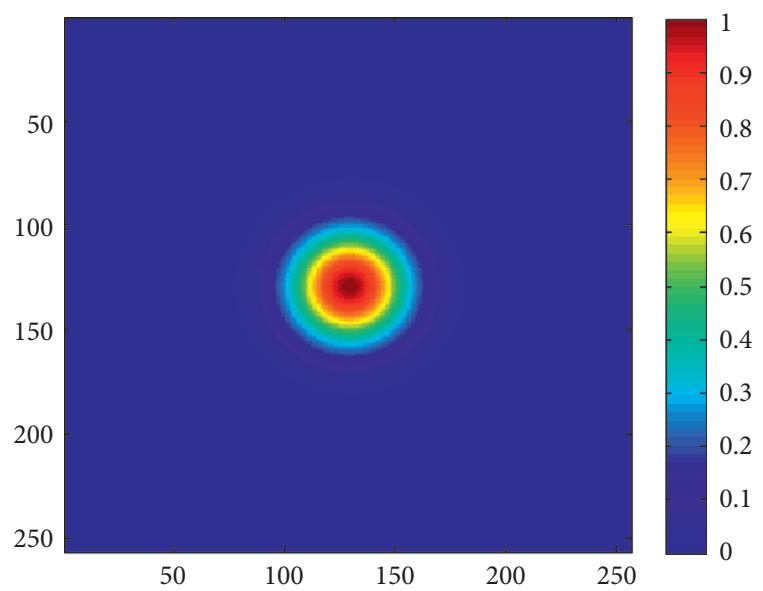

(b)

Figure 3: Initial Gaussian beam. (a) Spot pattern of initial Gaussian beam. (b) Stereograph of initial Gaussian beam.

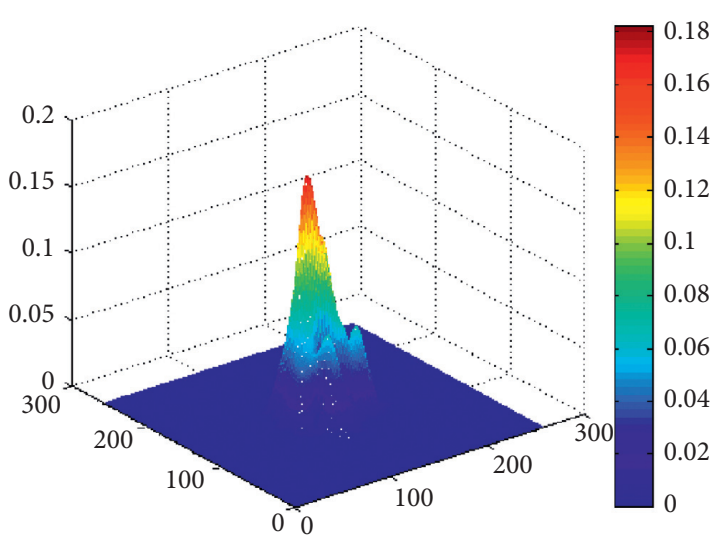

(a)

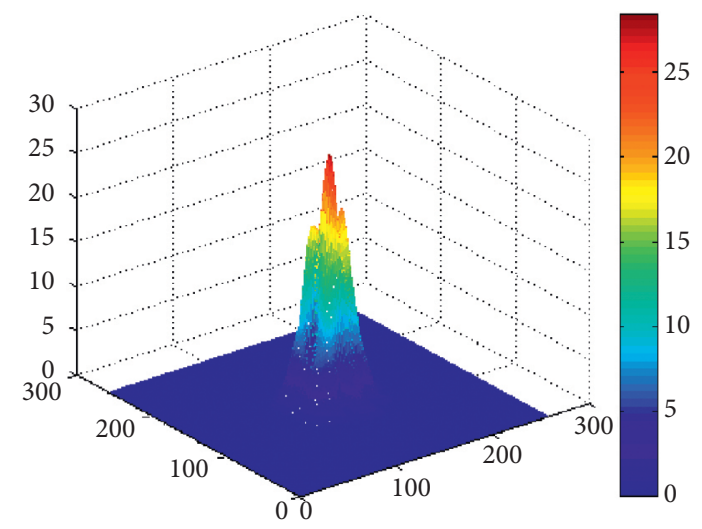

(c)

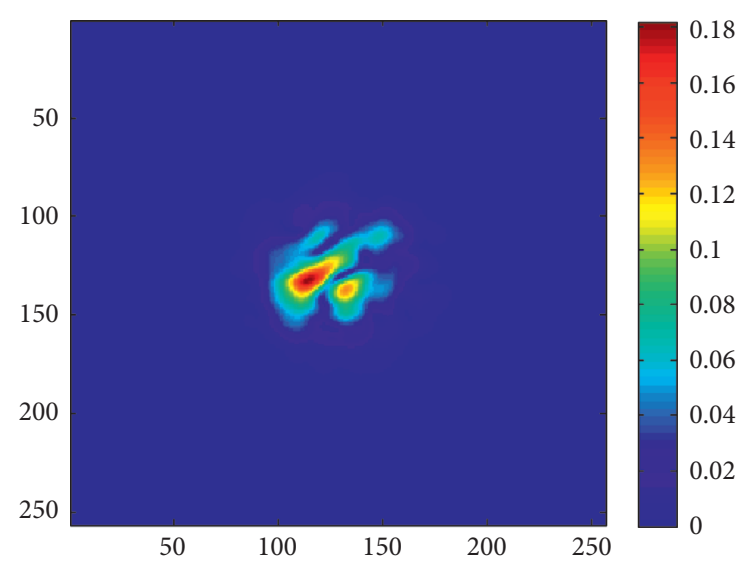

(b)

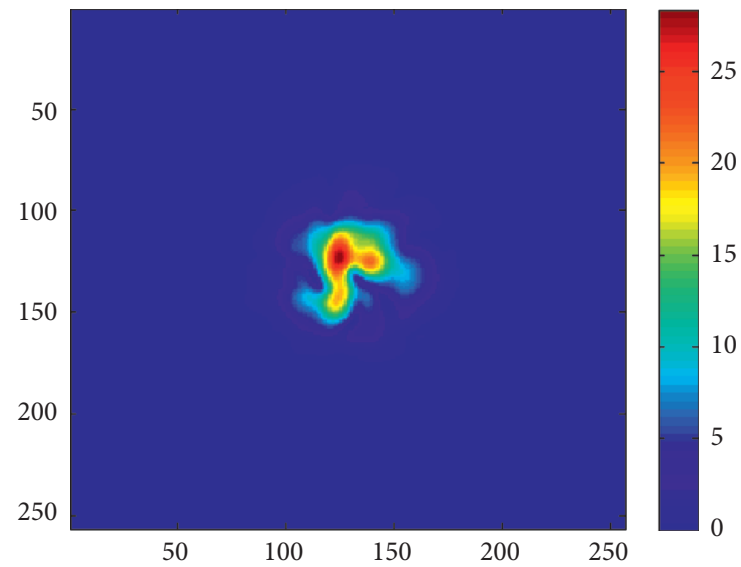

(d)

FIgURE 4: Continued. 


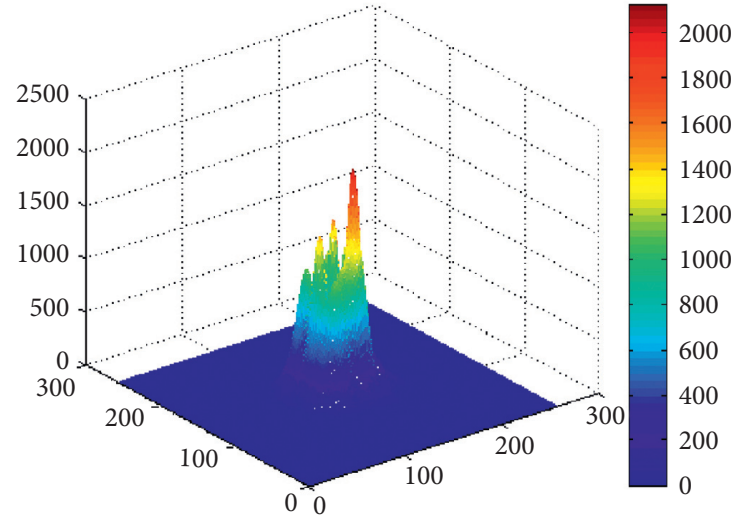

(e)

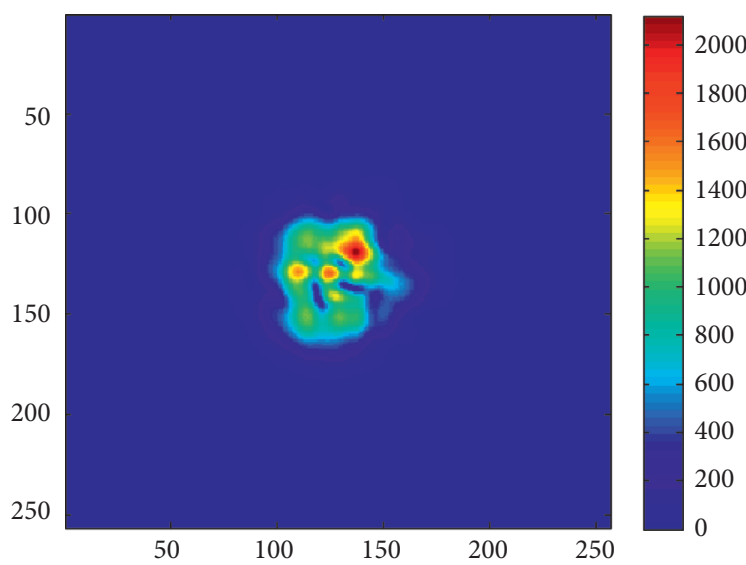

(f)

Figure 4: Gaussian beam after passing through the phase screen. (a) $C_{n}^{2}=2 \times 10^{-17} \mathrm{~m}^{-2 / 3}$, (b) $C_{n}^{2}=2 \times 10^{-17} \mathrm{~m}^{-2 / 3}$, (c) $C_{n}^{2}=2 \times 10^{-15} \mathrm{~m}^{-2 / 3}$, (d) $C_{n}^{2}=2 \times 10^{-15} \mathrm{~m}^{-2 / 3}$, (e) $C_{n}^{2}=2 \times 10^{-13} \mathrm{~m}^{-2 / 3}$, and (f) $C_{n}^{2}=2 \times 10^{-13} \mathrm{~m}^{-2 / 3}$.

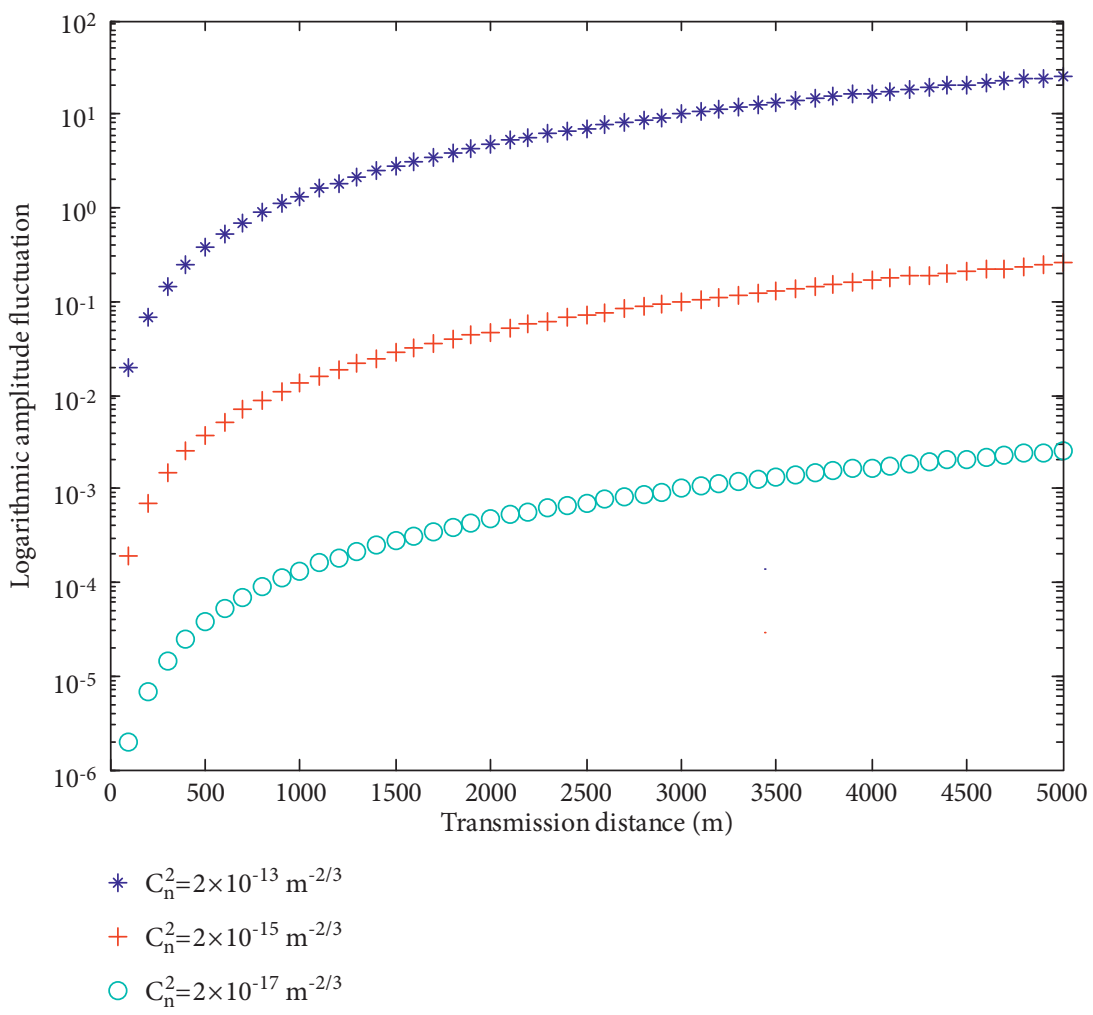

FIGURE 5: Variation of amplitude fluctuation with transmission distance.

From this, the relationship between bit error rate and light intensity fluctuation can be obtained [20,21]:

$$
\mathrm{BER}=\frac{1}{2}\left[\operatorname{erfc}\left(\frac{4}{\sqrt{2} \times 1.24 C_{n}^{2} k^{7 / 6} L^{11 / 6}}\right)\right] .
$$

Under different fluctuation conditions, the variation of logarithmic amplitude fluctuation and logarithmic intensity fluctuation with transmission distance are shown in Figures 5 and 6, and the relationship between bit error rate and logarithmic light intensity fluctuation variance is shown in Figure 7. In addition, the influence of transmission distance on bit error rate is also given, as shown in Figure Figure 8.

Figures 5 and 6 show the variation of logarithmic amplitude fluctuation and logarithmic intensity fluctuation with transmission distance under different intensity turbulence conditions. It can be clearly seen from the figures that when the transmission distance is constant, the stronger the turbulence intensity, the greater the fluctuation of logarithmic amplitude and logarithmic intensity. 


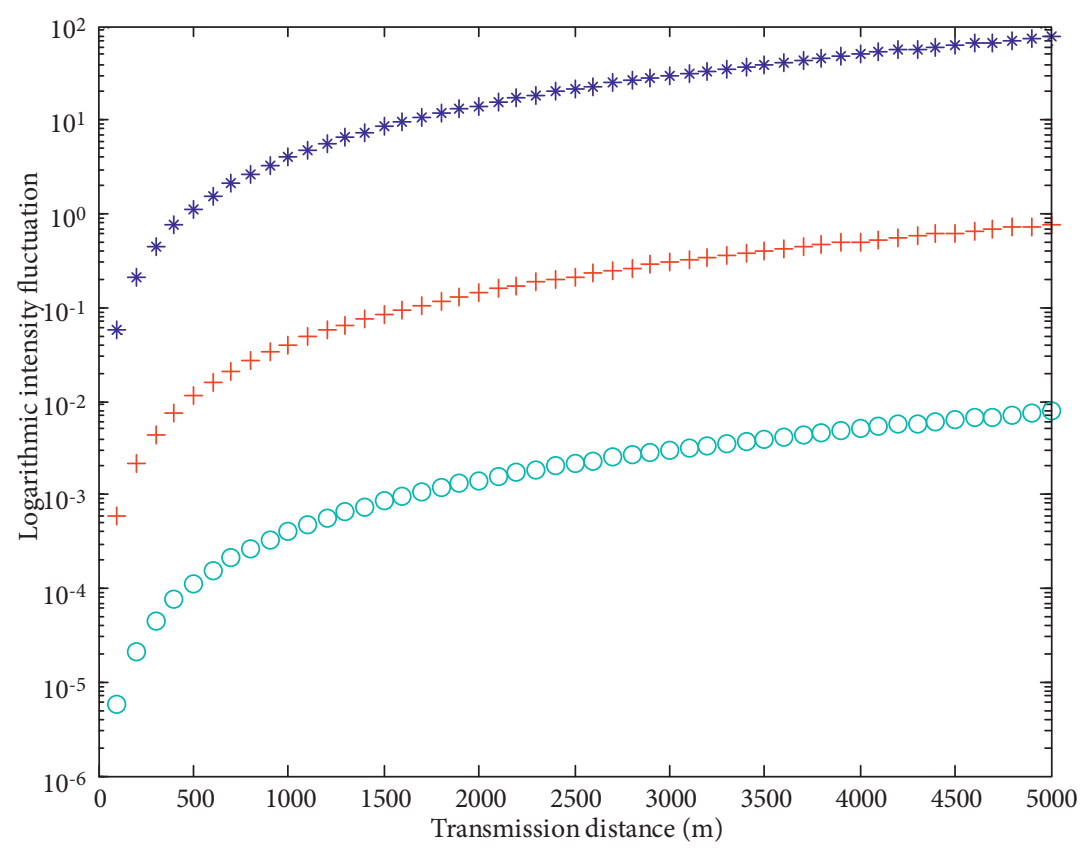

* $\mathrm{C}_{\mathrm{n}}^{2}=2 \times 10^{-13} \mathrm{~m}^{-2 / 3}$

$+\mathrm{C}_{\mathrm{n}}^{2}=2 \times 10^{-15} \mathrm{~m}^{-2 / 3}$

$\mathrm{C}_{\mathrm{n}}^{2}=2 \times 10^{-17} \mathrm{~m}^{-2 / 3}$

Figure 6: Variation of intensity fluctuation with transmission distance.

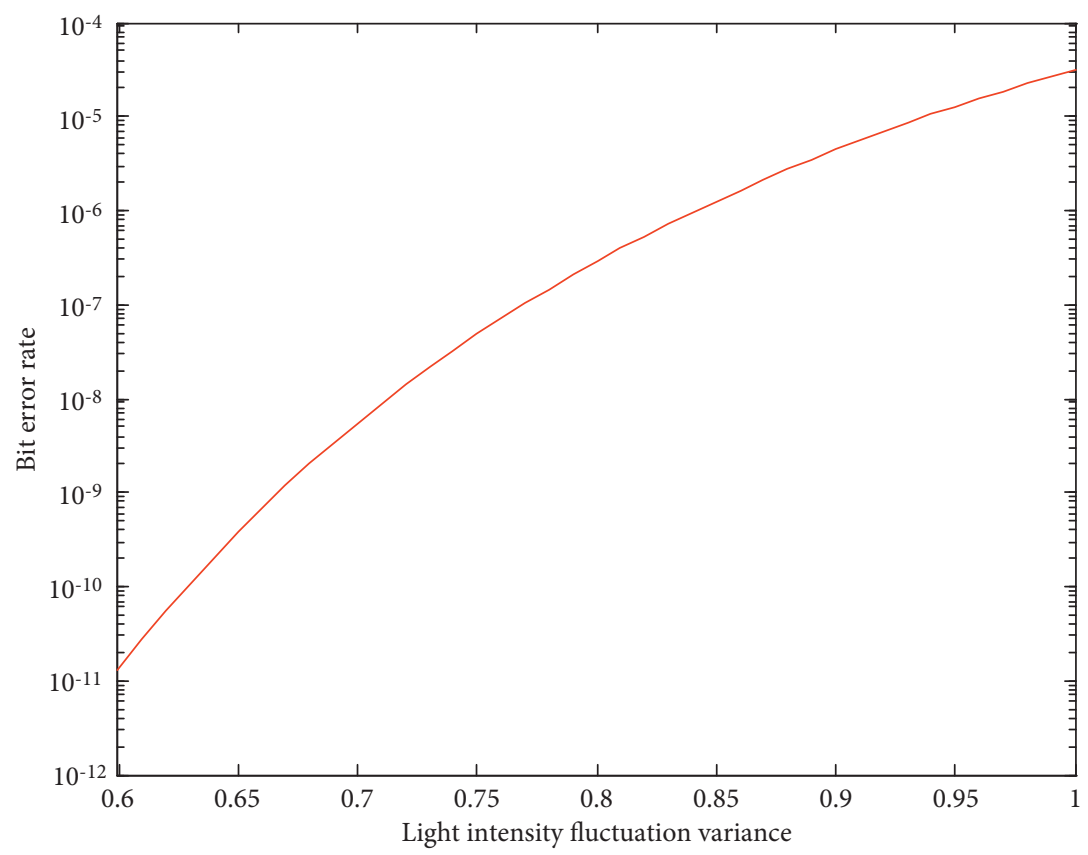

FIGURE 7: Variation of bit error rate with light intensity fluctuation.

In Free-Space Optical communication (FSO) system with a rate below $2.5 \mathrm{Gbps}$, the system's bit error rate is generally required to be below $10^{-9}$. It can be seen from Figure 7 that, under weak fluctuation conditions, for the atmospheric laser communication system's bit error rate less than the requirement, the light intensity fluctuation should be less than 0.67 . It can be seen from Figures 7 and 8 that when the transmission distance is constant, with the increase of turbulence intensity, the bit error rate rises quickly. For the bit error rate $10^{-9}$, when $C_{n}^{2}$ is equal to $2 \times 10^{-15} \mathrm{~m}^{-2 / 3}$, the effective distance of communication is close to $4.6 \mathrm{~km}$. With the increase of $C_{n}^{2}$, the effective distance of 


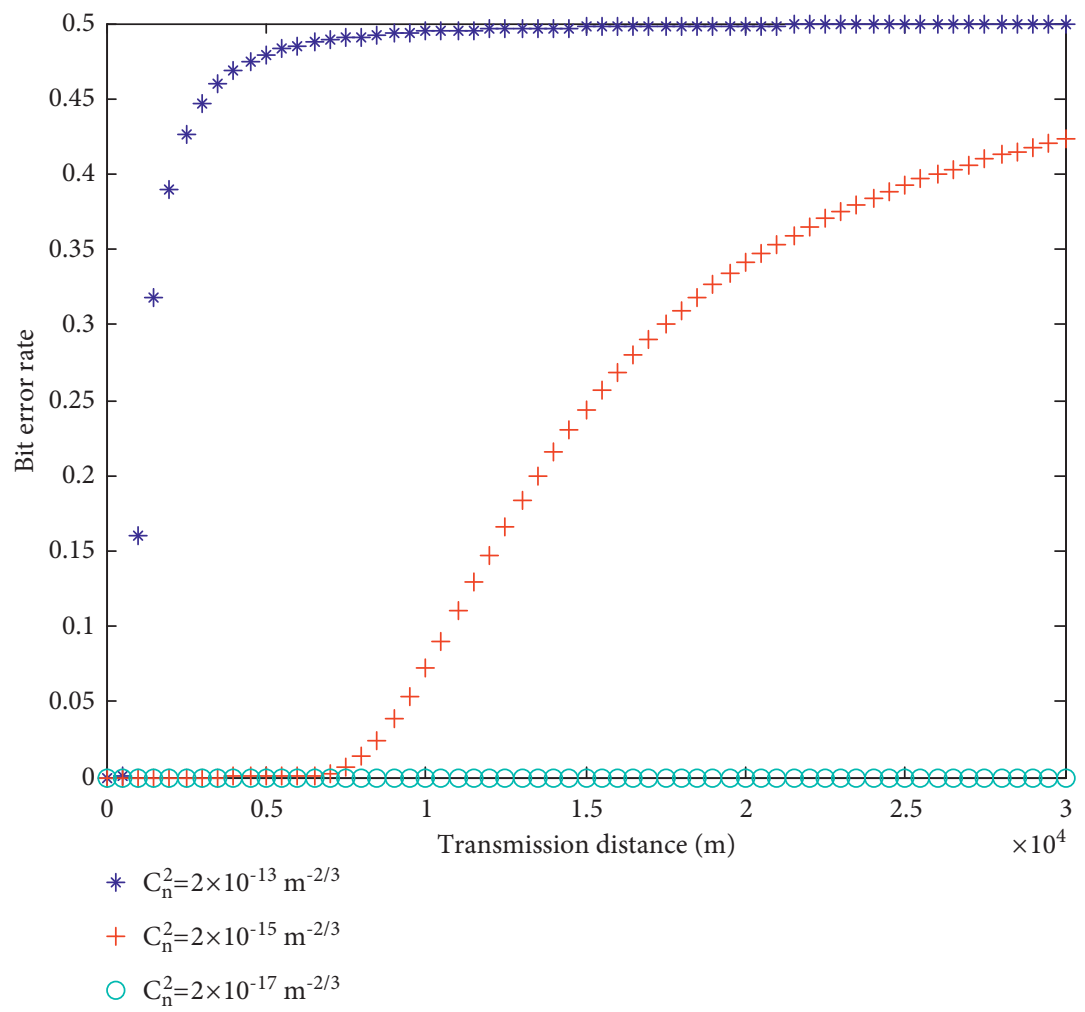

Figure 8: Variation of bit error rate with transmission distance.

transmission is getting smaller and smaller; when it reaches $2 \times 10^{-13} \mathrm{~m}^{-2 / 3}$, the effective distance of transmission reduced to about $0.37 \mathrm{~km}$.

\section{Conclusion}

By comparing the phase screens under atmospheric turbulence of different intensities, it is found that atmospheric turbulence of different intensities affects the propagation of Gaussian beams in terms of light intensity and phase fluctuations. The greater the intensity of the atmospheric turbulence is, the greater the light intensity of the Gaussian beam is affected, the more obvious the fluctuation of the light intensity, and the more obvious the tendency of the light spot to split into several pieces. In terms of phase fluctuations, the greater the intensity of the turbulence, the more intense the phase fluctuations of the Gaussian beam, the larger the difference of the light spot in the figure, and the more obvious the chromatic aberration, which puts forward higher and more specific requirements in the accuracy and reliability of laser transmission under atmospheric turbulence of different intensities. With the increase of turbulence intensity, the bit error rate rises quickly. With the increase of $C_{\mathrm{n}}{ }^{2}$, the effective distance of transmission is getting smaller and smaller; when it reaches $2 \times 10^{-13} \mathrm{~m}^{-2 / 3}$, the effective distance of transmission reduced to about $0.37 \mathrm{~km}$.

\section{Data Availability}

All datasets generated for this study are included within the article.

\section{Conflicts of Interest}

The authors declare that they have no conflicts of interest.

\section{Acknowledgments}

This work was supported by the National Key R\&D Program-Key Special Project of Inter-governmental S\&T Innovation Cooperation (2018YFE0199200), Key R \& D Program of Shaanxi Province (2019GY-081), and Key projects of Shaanxi Provincial Department of Education (20JY030).

\section{References}

[1] R. Frehlich, "Simulation of laser propagation in a turbulent atmosphere," Applied Optics, vol. 39, no. 3, pp. 393-397, 2000.

[2] H. Liu, Y. Pan, J. Cao, H. Wang, and Y. Zhou, "Adaptive neural network backstepping control of fractional-order nonlinear systems with actuator faults," IEEE Transactions on Neural Networks and Learning Systems, vol. 99, pp. 1-12, 2020.

[3] S. A. Ponomarenko, J. J. Greffet, and E. Wolf, "The diffusion of partially coherent beams in turbulent media," Optics Communications, vol. 208, no. 1, 2002.

[4] Y. Yang, W. Li, and H. Zhang, "A seven-parameter BRDF model with double-peak characteristic suitable for sandy soil," Mathematical Problems in Engineering, vol. 10, no. 3, 8 pages, Article ID 9398608, 2018.

[5] J. A. Fleck, J. R. Morris, and M. D. Feit, “Time-dependent propagation of high energy laser beams through the atmosphere," Applied Physics, vol. 10, no. 2, pp. 129-160, 1976. 
[6] B. L. McGlamery, "Restoration of turbulence-degraded images *," Journal of the Optical Society of America, vol. 57, no. 3, pp. 293-297, 1967.

[7] R. J. Noll, "Zernike polynomials and atmospheric turbulence *," Journal of the Optical Society of America, vol. 66, no. 3, pp. 207-211, 1976.

[8] E. P. Walner, "Optimal wave-front correction using slope measurements," Journal of the Optical Society of America, vol. 73, no. 12, pp. 1771-1776, 1983.

[9] K. W. Bowman and W. T. Rhodes, "Application of wavelets to wavefront reconstruction in adaptive optical systems," Adaptive Optics and Applications. International Society for Optics and Photonics, vol. 3126, pp. 288-299, 1997.

[10] L. C. Andrews and R. L. Phillips, Laserbeam Propagation Though Random Media, SPIE Optical Engineering Press, Bellingham, WA, USA, 2001.

[11] R. L. Fante, "Electromagnetic beam propagation in turbulent media," Proceedings of the IEEE, vol. 63, no. 12, pp. 1669-1692, 1975.

[12] Y. Jiang, J. Ma, L. Tan, S. Yu, and W. Du, "Measurement of optical intensity fluctuation over an $11.8 \mathrm{~km}$ turbulent path," Optics Express, vol. 16, no. 10, 2008.

[13] V. P. Aksenov, V. V. Dudorov, V. V. Kolosov, and V. Y. Venediktov, "Probability distribution of intensity fluctuations of arbitrary-type laser beams in the turbulent atmosphere," Optics Express, vol. 27, no. 17, 2019.

[14] R. Z. Rao, Light Propagation in the Turbulent Atmosphere, Anhui Science and Technology Press, Hefei, China, 2005.

[15] H. M. Zhang, The Research of Simulation of Laser Transmit in the Atmosphere Turbulence, Institute of Optics and Electronics of Chinese Academy of Sciences, Chengdu, China, 2005.

[16] D. Chen, Ke Xi-zheng, and Lu Zhang, "Laser intermodulation distortion and characteristic under the turbulence channel," Acta Photonica Sinica, vol. 45, no. 2, pp. 1-5, Article ID 0206007, 2016.

[17] Y. Baykal, "Signal-to-noise ratio reduction due to oceanic turbulence in oceanic wireless optical communication links," Optics Communications, vol. 427, pp. 44-47, 2018.

[18] B. Dima, "Free-space optical channel simulator for weakturbulence conditions," Applied Optics, vol. 31, 2015.

[19] Y. Xiang and M. Yao, "Free-space communications over exponentiated Weibull turbulence channels with nonzero boresight pointing errors," Optics Express, vol. 23, no. 3, 2015.

[20] J. C. Ricklin, S. M. Hammel, D. Frank, and S. Lachinova, "Atmospheric channel effects on free-space laser communication," Journal of Optical and Fiber Communications Reports, vol. 3, no. 2, 2006

[21] A. K. Majumdar, "Free-space laser communication performance in the atmospheric channel," Journal of Optical and Fiber Communications Reports, vol. 2, no. 4, 2005. 